\title{
Anti-obesity Effect of Fermented Persimmon Extracts via Activation of AMP-Activated Protein Kinase
}

\author{
Minhyeok Song, Goowon Yang, Tran Quynh Hoa, Hoang Dang Hieu, Ain Syafiza Mohd Amin, \\ Wonchae Choe, Insug Kang, Sung Soo Kim, and Joohun Ha* \\ Department of Biochemistry and Molecular Biology, Graduate School, College of Medicine, Kyung Hee University; \\ 26 Kyungheedae-ro, Dongdaemun-gu, Seoul 02447, Republic of Korea. \\ Received September 10, 2019; accepted December 3, 2019
}

There is significant cultivation of persimmon (Diospyros kaki) in East Asia, a plant whose fruit has abundant nutrients, including vitamins, polyphenols, and dietary fiber. Persimmon dietary supplements can benefit health by amelioration of diabetes, cardiovascular disease, and obesity. There are also persimmonbased beverages produced via fermentation, such as wines and vinegars, and increasing consumption of these products in East Asia. Although there is great interest in functional foods, the health effects of fermented persimmon extract (FPE) are completely unknown. We examined the effects of FPE on the metabolic parameters of mice fed a high-fat diet (HFD). Our results indicated that FPE supplementation led to an approx. $15 \%$ reduction of body weight, reduced abdominal and liver fat, and reduced serum levels of triglycerides, total cholesterol, and glucose. FPE also blocked the differentiation of murine 3T3-L1 pre-adipocyte cells into mature adipocytes. We suggest that gallic acid is a major bioactive component of FPE, and that AMPactivated protein kinase mediates the beneficial effects of FPE and gallic acid.

Key words fermented persimmon extract; anti-obesity effect; AMP-activated protein kinase; gallic acid; polyphenol

\section{INTRODUCTION}

The persimmon (Diospyros kaki) is a fleshy fibrous deciduous fruit that is widely cultivated in warm regions of East Asia. The global production of persimmon has gradually increased over time, and was 4.6 million tons in 2013, with $43 \%$ from China, $6 \%$ from Korea, and 5\% from Japan. ${ }^{1)}$ The fruit is highly nutritious, and contains various types of tannins, polyphenols, carotenoids, potassium, vitamins, and dietary fiber. ${ }^{2,3)}$ Several studies have demonstrated that consumption of persimmon helps to ameliorate various diseases, including cardiovascular disease, ${ }^{4)}$ diabetes ${ }^{5-8)}$ obesity, ${ }^{9-14)}$ and hypertension. ${ }^{15)}$ Despite its health-promoting potential, persimmon has several properties that prevent its more widespread consumption. First, it is difficult to store the fruits at room temperature and, because of the thin skin and very juicy flesh, they must be consumed within a few days after harvest. Second, many people in Western societies dislike the astringency of the fruit due to the abundance of tannins. For these reasons, several East Asian producers are developing persimmon into a variety of products, such as vinegar, beverages, and wines, through fermentation processes. ${ }^{16-19)}$ Fermentation is widely used to increase the shelf-life and nutritional value of foods, because many biochemical changes occur during fermentation that can affect the bioactivity and digestibility of these products. ${ }^{20)}$ For example, the content of bioactive phenolic compounds and flavonoids in legumes increases during fermentation because of bioconversion of conjugated phenolic compounds into free forms via microbial hydrolysis. Thus, fermented legumes have greater antioxidant activity than fresh legumes. ${ }^{21,22)}$

Although dietary supplementation with persimmon extracts can lower the plasma triglycerides (TGs) and cholesterol levels of laboratory animals with experimentally induced obesity, ${ }^{9-14)}$

* To whom correspondence should be addressed. e-mail: hajh@khu.ac.kr the underlying mechanisms are yet to be elucidated. Moreover, the effects of fermented persimmon extracts (FPE) on the metabolic parameters and the underlying mechanisms of these effects are essentially unknown.

In the present study, we examined the effect of a dietary FPE supplement on mice that were fed high-fat diets (HFDs) by measuring changes in body weight; plasma levels of glucose, TGs, and cholesterol; and liver steatosis. We then examined the possible mechanism of the effect of FPE by measuring the AMP-activated protein kinase (AMPK)-mediated differentiation of murine 3T3-L1 adipocytes and the effect of gallic acid, a major component of FPE whose level increases during fermentation. We further examined the novel mechanisms by which AMPK, which plays a central role in the regulation of glucose and lipid homeostasis, ${ }^{23-26)}$ may mediate the effects of FPE and gallic acid.

\section{MATERIALS AND METHODS}

Preparation of FPE FPE was obtained from Yangchon Persimmon Wine Farm Corporation (Nonsan City, Yangcheonmyeon, Korea). It was prepared by crushing persimmons and incubation with $2.5 \%$ tartaric acid, $1.5 \%$ citric acid, and $250 \mathrm{ppm}$ sulfite in sterile conditions. Then, 0.2\% Monascus purpureus (KCCM 60170, Korean Culture Center of Microorganisms, Seoul, South Korea) was added and the mixture was maintained at about $26^{\circ} \mathrm{C}$ for $10 \mathrm{~d}$. The temperature was then raised to $50^{\circ} \mathrm{C}$ for $3 \mathrm{~d}$ to induce alcoholic fermentation. After this initial fermentation, the extract was transferred into a new container and inoculated with pectin lyase (Pascal Biotech, Paris, France), and a red wine yeast and incubated at $26^{\circ} \mathrm{C}$ for 14d. Cellulase (Lot No. CTD1150106, Amano Enzyme Inc., Nagoya, Japan) was added, cells were incubated for another 
$30 \mathrm{~d}$, the yeast was removed, and the sediment was stirred ( $b \hat{a}-$ tonnage) for $50 \mathrm{~d}$. The FPE was then filtered and maintained at $18^{\circ} \mathrm{C}$ for 1 year and then evaporated on a rotary evaporator (Laborota 4000, Heidolph Instruments Inc., Schwabach, Germany) to remove the alcohol.

Animal Model of Obesity Animal studies were performed with the approval of the Kyung Hee University Animal Care and Use Committee (KHUASP(SE)-18-057). Threeweek old male C57BL/6N mice (Taconic Farms, Hudson, NY, U.S.A.) were housed at $21 \pm 2{ }^{\circ} \mathrm{C}$, with $50 \pm 5 \%$ relative humidity and a $12 \mathrm{~h}$ light $/ 12 \mathrm{~h}$ dark cycle. For adaptation to the laboratory environment, all mice received a normal diet (ND; D12450B, Research Diets, New Brunswick, NJ, U.S.A.), which provides $10 \%$ of calories as fat, for one week. Mice were then divided into three weight-matched groups (11 per group): the same normal diet (ND); a HFD, which provides $40 \%$ of calories as fat (D12492 HFD; Research Diets); and a HFD with + FPE $8 \%$ (w/w), which also provides $40 \%$ of calories as fat. Daily food intake was restricted to $2.7 \mathrm{~g}$ per mouse and the weights of all mice were recorded daily. At the end of the experimental period mice were deprived of food for $12 \mathrm{~h}$, and then anesthetized with diethyl ether prior to sacrifice. Blood was collected from the aorta into ethylenediaminetetraacetic acid (EDTA)-coated tubes. Mice were then dissected, and tissue weights were measured. All specimens were immediately frozen in liquid nitrogen and stored at $-80^{\circ} \mathrm{C}$ prior to analysis.

Quantitative Analysis of Phenolic Compounds HPLC (Jasco, Japan) analyses were performed with a Bondapak C18 $(10 \mu \mathrm{m}, 3.9 \times 300 \mathrm{~mm})$ column. The mobile phase contains $2 \%$ acetic acid (solvent $\mathrm{A}$ ) and $50 \%$ acetonitrile containing $0.5 \%$ acetic acid (solvent B). The injection volume was $20 \mu \mathrm{L}$, and the flow rate was adjusted to $0.8 \mathrm{~mL} / \mathrm{min}$. The column temperature was maintained at $40^{\circ} \mathrm{C}$. A gradient elution was performed over $70 \mathrm{~min}$ by varying the proportion of solvent $\mathrm{B}$ to solvent A, $10-80 \%$. The signal was detected at $280 \mathrm{nM}$. Gallic acid, catechin, epicatechin, gallocatechin, gallocatechingallate, and epicacatechin gallate were purchased from Sigma (SigmaAldrich, St. Louis, MO, U.S.A.) and used as standards for quantification.

Micro-Computed Tomography (CT) At the end of the experimental period but before dissection, transverse microCT images were taken of the L2 lumbar spine section, and three-dimensional (3D) images were reconstructed from visceral and subcutaneous fat areas of these images of the L2 to L5 lumbar spine sections.

Antibodies and Reagents Antibodies against phospho (Threonine 172)-AMPK $\alpha$, total AMPK $\alpha$, phospho (Serine 79)-acetyl-CoA carboxylase (ACC), and total ACC were purchased from Cell Signaling Technology (Danvers, MA, U.S.A.). Antibodies against fatty acid synthase (FAS), SREBP-1c, Peroxisome proliferator-activated receptor gamma $(\mathrm{PPAR} \gamma), \mathrm{CCAAT} / \mathrm{enhancer}$-binding protein alpha $(\mathrm{C} / \mathrm{EBP} \alpha)$, $\beta$-actin, and horseradish peroxidase (HRP)-conjugated secondary antibodies were purchased from Santa Cruz Biotechnology (Dallas, TX, U.S.A.). All cell culture media and supplements were obtained from Thermo Fisher Scientific (Waltham, MA, U.S.A.).

Cell Culture and Differentiation The murine 3T3-L1 adipocyte cell line was maintained in Dulbecco's modified Eagle's medium (DMEM), supplemented with 10\% fetal bo- vine serum (FBS) and antibiotics, and cultured at $37^{\circ} \mathrm{C}$ in $95 \%$ air with $5 \% \mathrm{CO}_{2}$. To induce differentiation, the cells were cultured until confluent. Differentiation was then induced by changing the medium to DMEM supplemented with $10 \%$ FBS plus a mixture of hormones $(0.5 \mathrm{mM}$ IBMX, $1 \mu \mathrm{M}$ dexametha sone, and $5 \mu \mathrm{g} / \mathrm{mL}$ insulin). After $48 \mathrm{~h}$, the differentiation medium was replaced with DMEM supplemented with $10 \%$ FBS and $5 \mu \mathrm{g} / \mathrm{mL}$ insulin and different concentrations of FPE and gallic aicd were added. After $8 \mathrm{~d}$, the mature adipocytes, which were round and had many oil droplets, were harvested for measurements.

Transfection of AMPKa1 Wild-Type (WT) and Dominant Negative (DN) Form into 3T3-L1 Pre-adipocyte 3T3-L1 pre-adipocyte were seeded on a six-well plate (Corning, NY, U.S.A.). After the cell density reached $60 \%$, cells were transfected with expression vectors pcDNA $3.1(+)$ for the WT or DN form of HA-tagged AMPK $\alpha 1$ for $24 \mathrm{~h}$ using Lipofectamine 3000 (Invitrogen, CA, U.S.A.) according to the manufacturer's instructions. Empty vector transfected cells were used as a negative control. Then, cells were cultured for $7 \mathrm{~d}$ in the presence of G418 selection $(600 \mathrm{mg} / \mathrm{mL})$ and these cells were used for differentiation. As the results, the percentage of cells expressing AMPK WT and DN increased to $80 \%$.

Oil Red O Staining The mature 3T3-L1 cells were washed twice with phosphate-buffered saline (PBS), fixed with $10 \%$ formaldehyde for $90 \mathrm{~min}$ at room temperature, washed with $60 \%$ isopropanol, and then dried completely. The fixed cells were then stained with Oil Red $\mathrm{O}$ for $1 \mathrm{~h}$ at room temperature, and then washed twice with distilled water. Lipid droplets were then observed by light microscopy and images were recorded (Olympus, Tokyo, Japan). Then 100\% isopropanol was added to each well to extract the Oil Red O dye, and absorbance was measured at $510 \mathrm{~nm}$ using a spectrophotometer (BioTek Instruments, Inc., Winooski, VT, U.S.A.).

Immunoblot Analysis Total proteins from mature 3T3-L1 cells were extracted in RIPA lysis buffer $(50 \mathrm{mM}$ Tris- $\mathrm{HCl}$ pH 7.4, $150 \mathrm{mM} \mathrm{NaCl}, 1 \mathrm{mM}$ EDTA, 1\% Triton X-100, 0.1\% sodium dodecyl sulfate (SDS), $0.5 \%$ sodium deoxycholate, $5 \mathrm{mM}$ sodium fluoride, $2 \mathrm{mM}$ sodium orthovanadate, $1 \mathrm{mM}$ phenylmethylsulfonyl fluoride (PMSF), and a protein inhibitor cocktail). The protein concentration of the lysate was determined using the Bradford assay. For immunoblotting, cellular proteins were separated by SDS-polyacrylamide gel electrophoresis (PAGE) and then transferred onto nitrocellulose membranes. The membranes were blocked with Tris-buffered saline (TBS) containing Tween $20(10 \mathrm{mM}$ Tris- $\mathrm{HCl} \mathrm{pH} 7.4$, $150 \mathrm{mM} \mathrm{NaCl}$, and $0.1 \%$ Tween 20 ) and $5 \%$ nonfat dry milk, and incubated overnight at $4{ }^{\circ} \mathrm{C}$ with different primary antibodies diluted in blocking buffer. The membranes were then washed and incubated with the appropriate secondary antibodies for $1 \mathrm{~h}$ at room temperature. HRP-conjugated secondary antibodies were detected using enhanced chemiluminescence (ECL) reagents.

RT-PCR Total RNA was extracted from mature 3T3-L1 cells using the TRIzol reagent, and cDNA was synthesized by RT from $1 \mu \mathrm{g}$ of total RNA using a cDNA Synthesis Kit, according to the manufacturer's instructions (Enzynomics, Daejeon, Korea). The primer pairs used for the PCR are as follows: Acc, 5'-GTT TGG TCG TGACTGCTC TG-3' (forward) and 5'-AGT TCG AAA GTC ACC CCGAA-3' (reverse); Fas, 5'-TCA GCT ATGAAGCAA TTGTG-3' (for- 
ward) and 5'-GAA TCA TCAAAGGATCTGCA-3' (reverse); Srebp-1c, 5'-AGCCAGGTCAAA GCCCAG-3' (forward) and 5'-CCCATA GACAAA GAGAAGAG-3' (reverse); Ppar $\gamma$, 5'-TGC AGG AGCAGA GCAAAGA-3' (forward) and 5'-TCA TCA GGGAGGCCA GCA TC-3' (reverse); C/Ebpo, 5'-ACC TGCCGCCCCGGA G-3' (forward) and 5'-GGCCAGGTG CGCGGGAGA CG-3' (reverse); $\beta$-actin, 5'-CTG TGC TGT CCC TGT ATGCCTC-3' (forward) and 5'-CCA GAC AGC ACTGTG TTGGC-3' (reverse). The following thermos-cycling regimen was used: $2 \mathrm{~min}$ at $95^{\circ} \mathrm{C}, 25$ cycles of $95^{\circ} \mathrm{C}$ for $30 \mathrm{~s}$, $55^{\circ} \mathrm{C}$ for $30 \mathrm{~s}$, and $72^{\circ} \mathrm{C}$ for $30 \mathrm{~s}$ and a final $2 \mathrm{~min}$ at $72^{\circ} \mathrm{C}$. PCR products were analyzed by agarose gel electrophoresis using $1 \%$ gels containing ethidium bromide. RT-PCR data were normalized to the $\beta$-actin level in each sample.

Histological Analysis Liver tissues from the three groups of mice (ND, HFD, and HFD + FPE) were perfused with $\mathrm{PBS}$, frozen in liquid nitrogen immediately, and then stored at $-80^{\circ} \mathrm{C}$. Then, $4 \mu \mathrm{m}$ sections were prepared using a cryostat microtome (Leica, Wetzlar, Germany), followed by mounting on gelatin-coated glass slides. After fixing with $4 \%$ paraformaldehyde, sections were stained with hematoxylin and eosin (H\&E) for microscopic assessment (Olympus). The frozen liver sections were stained with Oil Red $\mathrm{O}$ for histological examination of fat droplets.

Biochemical Analysis The concentrations of blood glucose, high density lipoprotein (HDL)-cholesterol, low density lipoprotein (LDL)-cholesterol, and TGs were determined using an Accu-Chek Active Kit (Roche Diagnostics, GmbH, Germany) and an automated analyzer (SMARTLAB, Mannheim, Germany).

Statistical Analysis The experimental results are expressed as the mean \pm standard error of the mean (S.E.M.) of at least three independent experiments. The difference between two means was analyzed using Student's $t$-test and considered statistically significant when $p<0.05$.
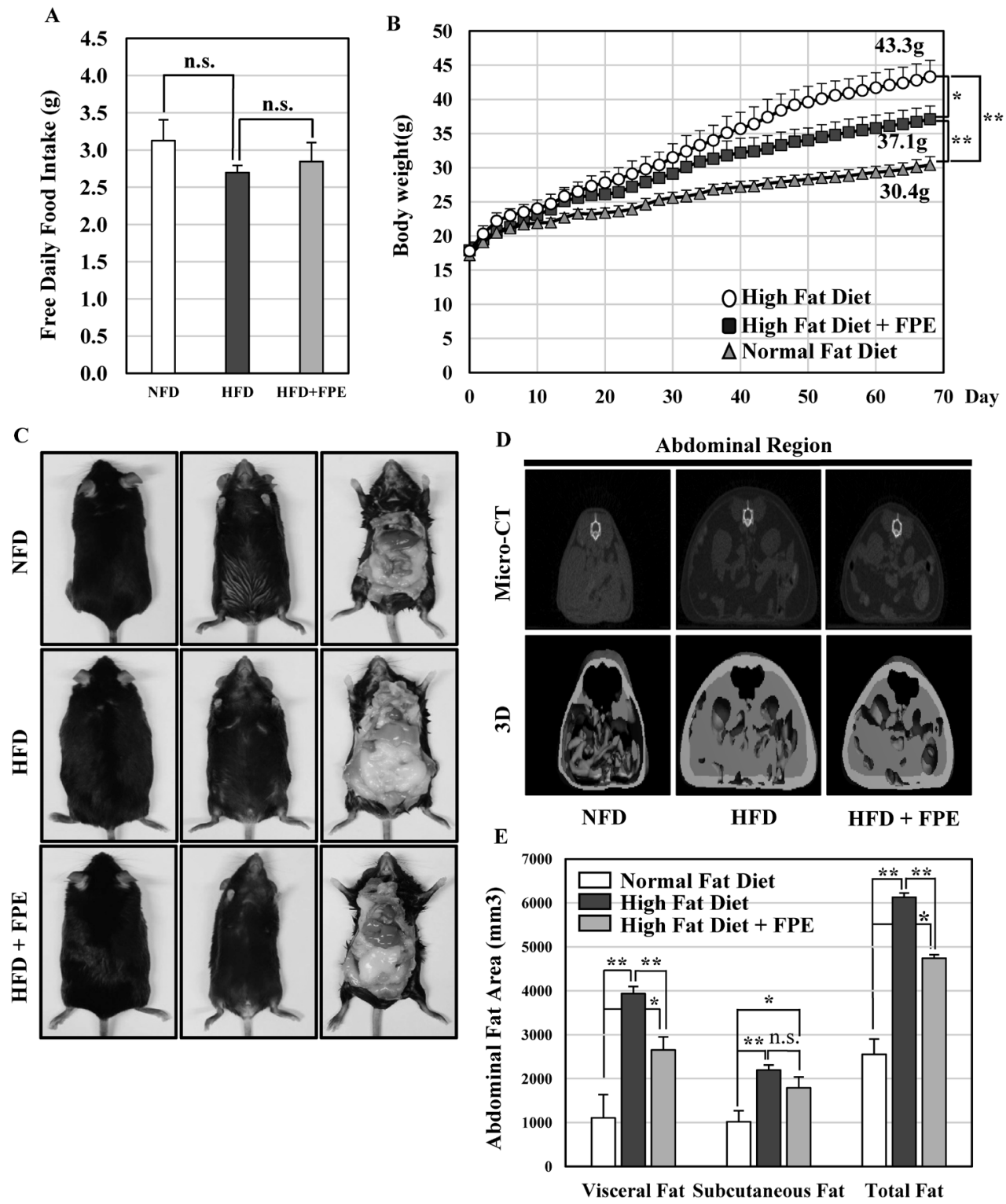

Fig. 1. FPE Reduces Body Weight, Visceral Fat, and Total Fat in Mice Fed HFDs

(A) Average daily food intake of mice in each group when they were allowed free access to different diets for 1 week. (B) Average weight of mice under restrictedintake conditions $(2.7 \mathrm{~g} / \mathrm{d})$ for 10 weeks. (C) Overall appearance and the abdominal regions. (D) Micro-CT images (upper) and 3D images (lower) of the abdominal regions. (E) Visceral, subcutaneous, total fat areas in the abdominal 3D images. Bars represent mean \pm standard deviation (S.D.) $(* p<0.05, * * p<0.01)$. 


\section{RESULTS}

FPE Reduces Body Weight and Abdominal Fat of Mice Receiving HFDs We investigated the effect of FPE on the body weight of mice by feeding them one of three diets (11 per group): normal diet (ND, 10\% of calories as fat), high-fat diet (HFD, $40 \%$ of calories as fat), and HFD with $8 \%(w / w)$ FPE (HFD + FPE). When mice were allowed free access to food for 1 week, the average intake was $3.1 \mathrm{~g}$ for the ND group, $2.7 \mathrm{~g}$ for the HFD group, and $2.8 \mathrm{~g}$ for the HFD + FPE group (Fig. 1A). Because unequal food intake by the different groups could affect the results, we restricted the daily food supply to $2.7 \mathrm{~g}$ per mouse in all three groups, and monitored their body weights for 10 weeks. The weight of HFD mice increased more rapidly than ND mice, but mice receiving HFD + FPE had a mean body weight $15 \%$ lower than HFD mice (Fig. 1B). Anatomical examinations indicated that the HFD + FPE mice had significantly less abdominal fat than HFD mice (Fig. 1C). Transverse micro-CT and 3D images indicated reduced visceral fat and total fat mass of HFD + FPE mice compared to HFD mice, although the two groups had similar subcutaneous fat masses (Figs. 1D, E). These results indicate that FPE has a strong anti-obesity effect.

FPE Reduces Fat in the Abdomen and Liver and Serum Levels of TGs, Cholesterol, and Glucose We also examined the effect of FPE on the major organs after sacrifice of mice on day 70 . The results indicated that HFD + FPE mice had $27 \%$ less abdominal fat and $16 \%$ less liver mass than HFD mice (Fig. 2A). Oil Red O and H\&E staining of liver tissues showed that HFD + FPE mice had significantly less hepatic steatosis than HFD mice (Fig. 2B). Serum analysis showed that HFD + FPE mice had lower serum levels of total cholesterol (Fig. 3A), TGs (Fig. 3B), fasting blood glucose (Fig. 3C), HDL-cholesterol (Fig. 3D), and LDL-cholesterol (Fig. 3E) relative to HFD mice. These results highlight the
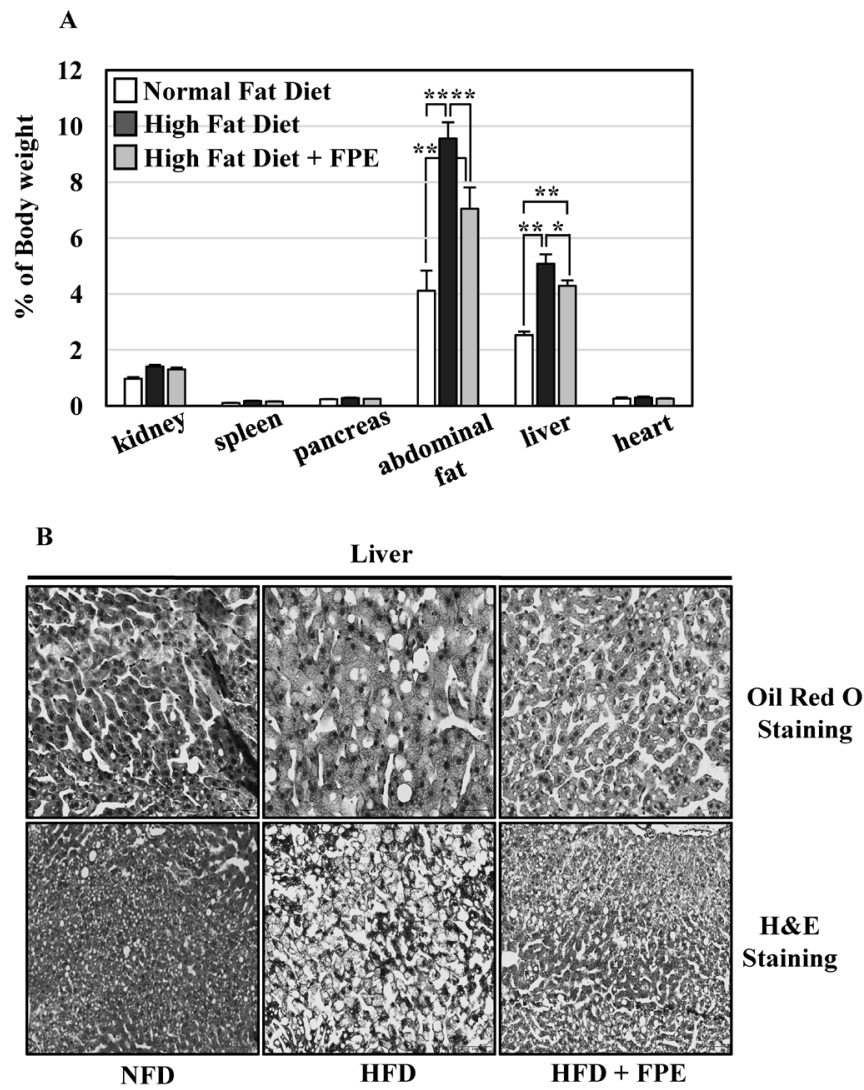

Fig. 2. FPE Reduces the Amount of Abdominal Fat, Liver Mass, and Fat Content of Mice Fed HFDs

(A) The weight of each organ was normalized to body weight. Bars represent mean \pm S.D. $(* p<0.05, * * p<0.01)$. (B) Histological analysis of liver tissue using Oil Red O staining (upper) and H\&E staining (lower). Magnification: $1000 \times$.
A
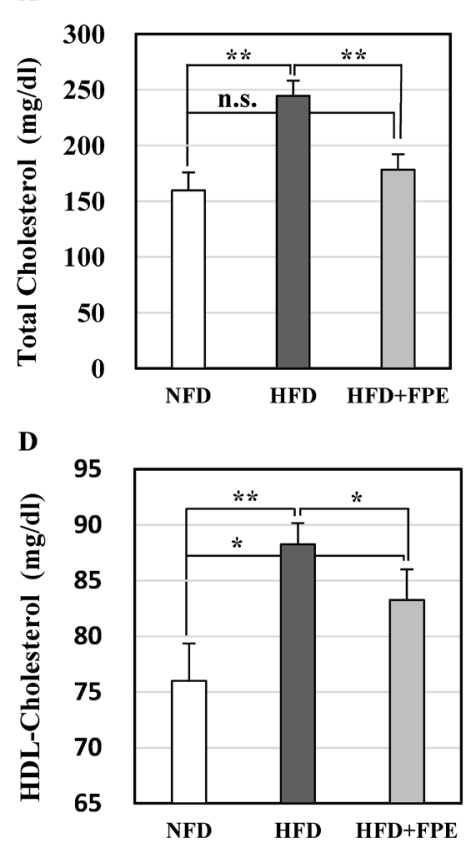

B
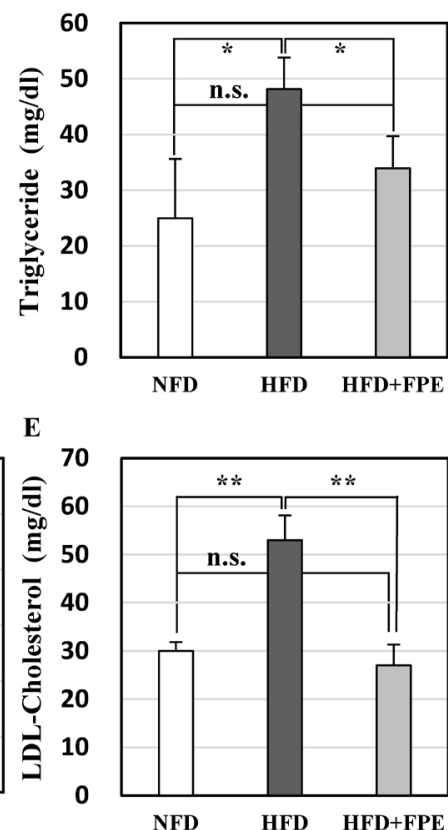

C

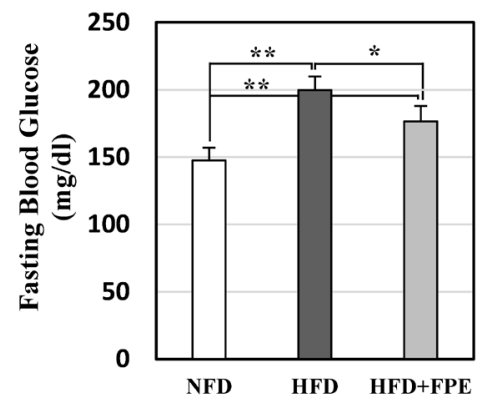

Fig. 3. FPE Reduces Blood Levels of Lipids and Fasting Glucose in Mice Fed HFDs

After sacrifice, the levels of total cholesterol (A), triglycerides (B), fasting blood glucose (C), HDL-cholesterol (D), and LDL-cholesterol (E) were measured. Bars represent mean \pm S.D. $\left({ }^{*} p<0.05,{ }^{* *} p<0.01\right)$. HDL, high-density lipoprotein; LDL, low-density lipoprotein. 
A

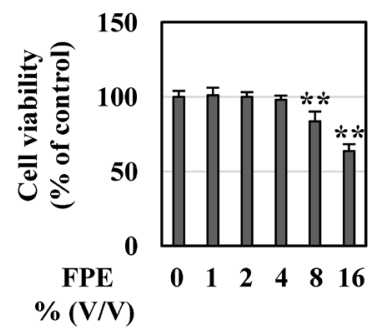

B

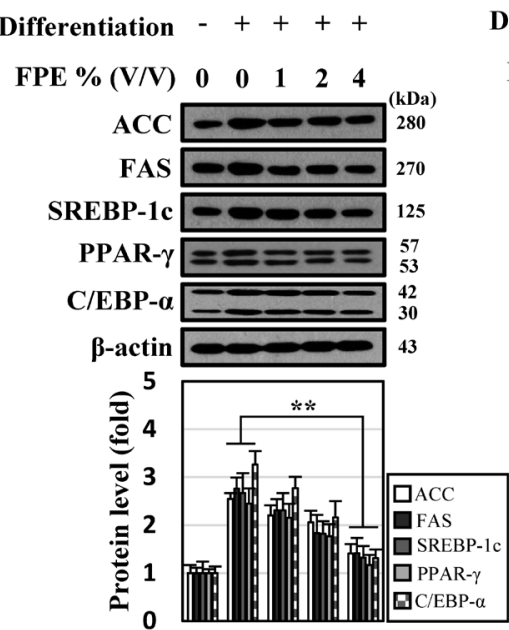

C

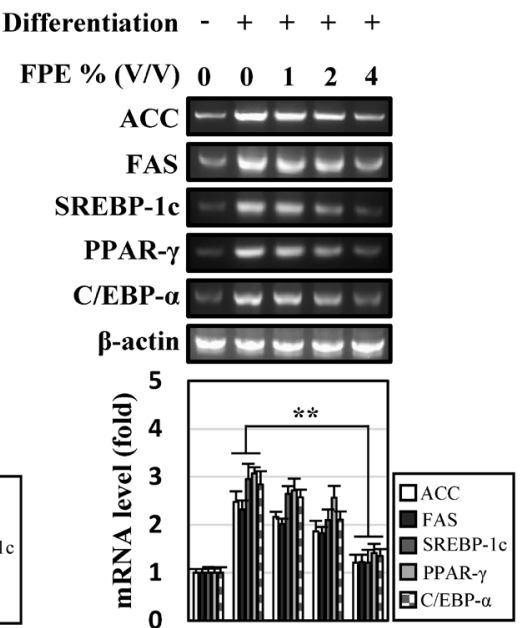

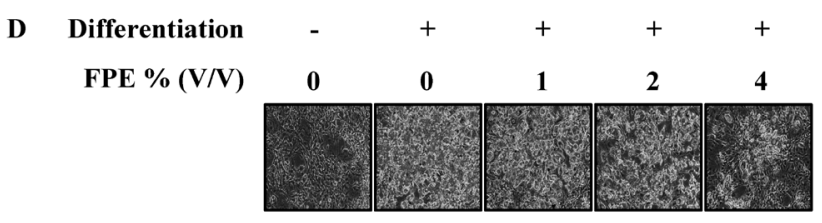

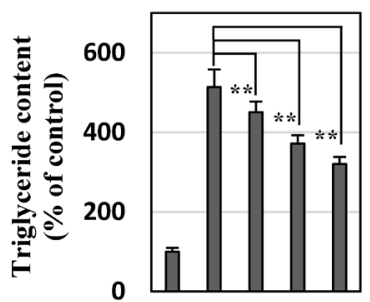

Differentiation - +++

FPE \% (V/V) $00 \begin{array}{lllllll}0 & 0 & 2 & 4\end{array}$

Fig. 4. FPE Reduces Fat Production and Differentiation of 3T3-L1 Adipocytes

(A) 3T3-L1 pre-adipocytes were treated with different concentrations of FPE for 48h and cell viability was measured using the MTT (3-[4,5-dimethylthiazol-2-yl]-2,5diphenyltetrazolium bromide) assay. (B, C) Different concentrations of FPE were added during adipocyte differentiation, and the levels of proteins and mRNAs involved in fatty acid synthesis were then analyzed by Western blotting and RT-PCR. (D) Under identical conditions, intracellular fat droplets were stained with Oil-Red O on day 8 , and the total fat content was determined by measurement of absorption at $510 \mathrm{~nm}$. Bars represent mean \pm S.D. $(* p<0.05$, $* * p<0.01)$.

Table 1. Major Polyphenolic Compounds Present in Persimmon Extracts before and after Fermentation

\begin{tabular}{lccc}
\hline \hline Name & Molecular weight & Before fermentation $(\mu \mathrm{g} / \mathrm{mL})$ & After fermentation $(\mu \mathrm{g} / \mathrm{mL})$ \\
\hline Gallic acid & 170.12 & 48 & 89.5 \\
(+)-Catechin & 290.27 & 9 & 3.1 \\
(-)-Epicatechin & 290.27 & 8 & 1.9 \\
(-)-Gallocatechin & 306.27 & 1 & 0 \\
(-)-Gallocatechin gallate & 458.37 & 9 & 0 \\
Epicatechin gallate & 442.37 & 11 & 1.6 \\
\hline
\end{tabular}

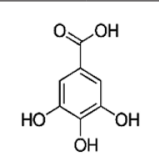

Gallic acid

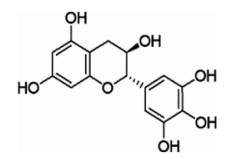

$(-)$-Gallocatechin

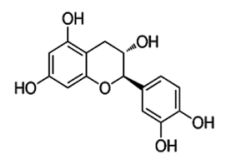

$(+)$-Catechin

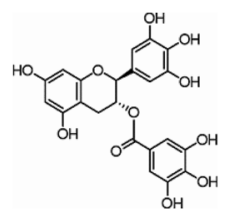

(-)- Gallocatechin gallate

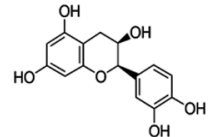

(-)-Epicatechin

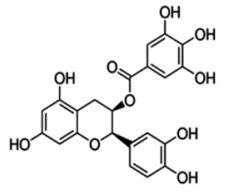

Epicatechin gallate specific anti-obesity properties of FPE.

FPE and Gallic Acid Inhibit Fatty Acid Synthesis and Differentiation of 3T3-L1 Adipocytes We next examined the possible molecular mechanism of FPE by determining its effect on the biochemical properties of murine 3T3-L1 adipocytes. FPE had no cytotoxic effects when the concentration 


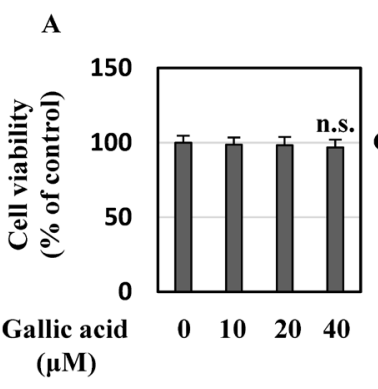

B

Differentiation - ++++

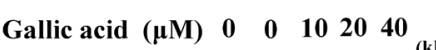
$\mathrm{ACC}-\infty-280$

FAS $-6-270$ SREBP-1c $-20-125$ PPAR- $\gamma$ =三三三 57 C/EBP- $\alpha$ 二三ニ二 ${ }_{30}^{42}$ $\beta$-actin 43

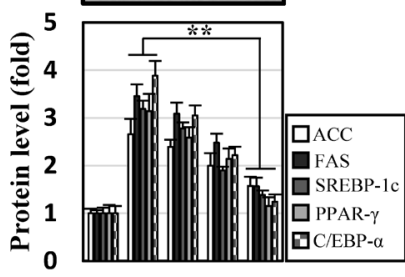

C

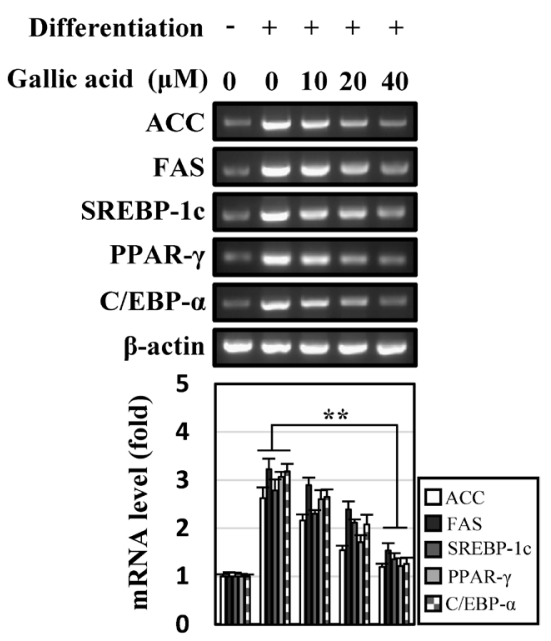

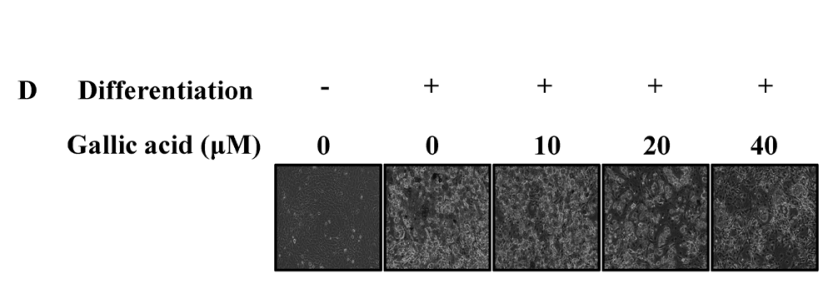

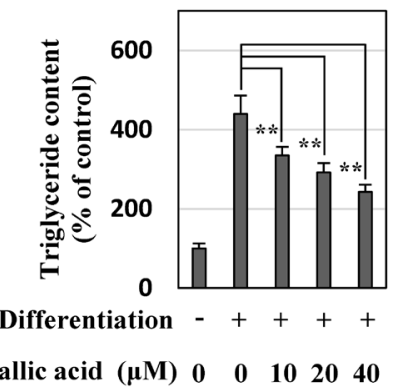

Fig. 5. Gallic Acid Reduces Fat Production and Differentiation of 3T3-L1 Adipocytes

(A) 3T3-L1 pre-adipocytes were treated with different concentrations of gallic acid for $48 \mathrm{~h}$ and cell viability was measured using the MTT assay. (B, C) Different concentrations of gallic acid were added during adipocyte differentiation, and the levels of proteins and mRNAs involved in fatty acid synthesis were then analyzed by Western blotting and RT-PCR. (D) Under identical conditions, intracellular fat droplets were stained with Oil-Red O on day 8 and total fat content was determined by measurement of absorption at $510 \mathrm{~nm}$. Bars represent mean \pm S.D. $(* p<0.05, * * p<0.01)$.

was $4 \%(\mathrm{v} / \mathrm{v})$ or less (Fig. 4A). Thus, we examined the effects of different levels of FPE (0 to 4\%) on the expression of genes involved in fatty acid synthesis and adipocyte differentiation. FAS and ACC play major roles in fatty acid synthesis, and sterol regulatory element-binding protein-1c (SREBP-1c) is a key transcription factor for fatty acid synthesis-related genes. $\operatorname{PPAR} \gamma$ and $\mathrm{C} / \mathrm{EBP} \alpha$ play important roles in inducing adipocyte differentiation. Our Western blotting (Fig. 4B) and mRNA analysis (Fig. 4C) indicated that FPE inhibited the expression of these genes in a dose-dependent manner. In agreement, Oil-Red $\mathrm{O}$ staining also indicated that FPE reduced lipogenesis in a dose-dependent manner (Fig. 4D).

HPLC analysis of the persimmon extracts before fermentation indicated the presence of various polyphenols, including gallic acid, catechin, epicatechin, with an especially high level of gallic acid (Table 1). Interestingly, the portion of gallic acid increased after fermentation (Table 1). Thus, we performed the same experiments reported in Fig. 4, but used gallic acid rather than FPE. A gallic acid level of $40 \mu \mathrm{M}$ had no evident cytotoxicity to 3T3-L1 adipocytes (Fig. 5A). Similar to FPE, gallic acid inhibited genes involved in fatty acid synthesis and adipocyte differentiation (Figs. 5B, C) and reduced lipogenesis of 3T3-L1 adipocytes in a dose dependent manner (Fig. 5D).

FPE and Gallic Acid Inhibit Fatty Acid Synthesis in 3T3-L1 Cells by AMPK Activation We next examined the effect of FPE and gallic acid on AMPK, which plays a central role in the regulation of glucose and lipid homeo- stasis. Our Western blotting experiments indicated that FPE activated AMPK in a dose-dependent manner, as evidenced by the increase of Thr172-phosphoyrlated AMPK and Ser79phosphorylated ACC, an intracellular substrate of AMPK (Fig. 6A). Compound $\mathrm{C}$, an AMPK inhibitor, significantly blocked the effects of FPE on gene expression involved in fatty acid synthesis (Fig. 6B). The results were similar when endogenous AMPK activation by FPE was blocked by expression of an AMPK dominant negative (DN) form, whereas expression of the wild-type AMPK potentiated the effect of FPE (Fig. 6C). These results indicate that AMPK has an important role in the anti-lipogenesis effect of FPE. The results were similar when we used gallic acid instead of FPE (Fig. 7). Thus, our results suggest that the FPE inhibits the synthesis of fatty acids by activating AMPK and downregulating the genes involved in fatty acid synthesis, and that gallic acid is the major bioactive component of FPE.

\section{DISCUSSION}

Fermentation can improve the properties of foods, such as bioactivity and digestibility, ${ }^{20)}$ and the amount of phenolic compounds and antioxidant activity also increase during fermentation. ${ }^{21,22)}$ There are ongoing efforts in East Asia to increase the commerciality of various products of persimmon that are produced through fermentation, but the biological effects of FPE and the underlying mechanisms are yet to be 
A
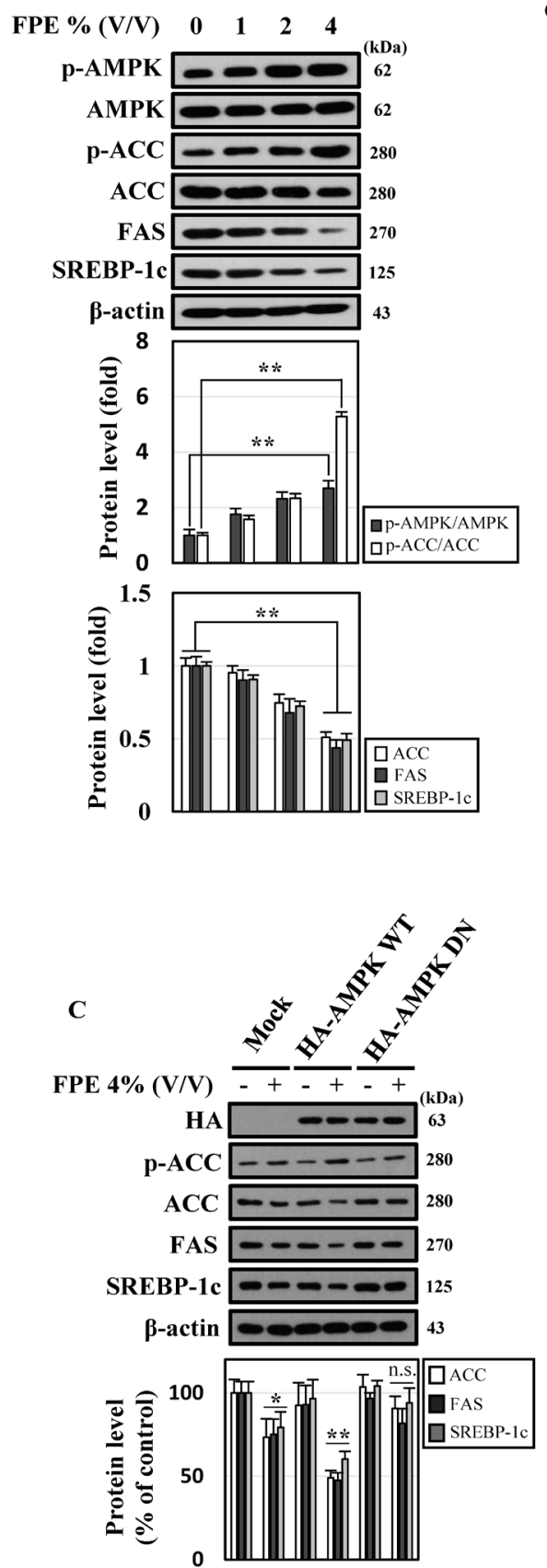

B FPE 4\%(V/V) - - + +

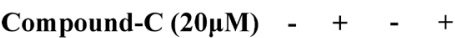

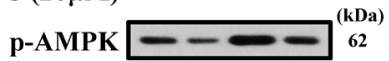

АMPK $--\infty$

p-ACC $---{ }_{280}$

$\mathrm{ACC}-\square$

FAs $---{ }_{270}$

SREBP-1c -2

$\beta$-actin $\longrightarrow 43$
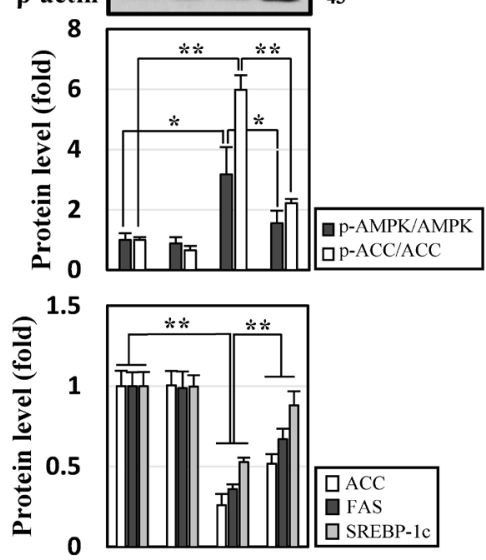

Fig. 6. FPE Inhibits the Expression of Genes Involved in Fatty Acid Synthesis by Activation of AMPK in 3T3-L1 Adipocytes

(A, B) Different concentrations of FPE were added into 3T3-L1 cell in the absence or presence of compound C (AMPK inhibitor). (C) 3T3-L1 cell were transfected with the expression vectors for wild type (WT) or dominant negative (DN) form of HA-tagged AMPK and then incubated with FPE for $24 \mathrm{~h}$. Under the conditions, western blots were performed. Bars represent mean \pm S.D. $(* p<0.05, * * p<0.01)$.

elucidated. The present study demonstrated that FPE has potent anti-obesity effects in mice (Figs. 1 to 3 ). It is intriguing that during persimmon fermentation, the content of free gallic acid increased, whereas the content of phenolic compounds containing the gallic acid moiety, such as (-)-gallocatechin gallate and epicatechin gallate, decreased (Table 1). It is not clear at this point whether (-)-gallocatechin gallate and epicatechin gallate are the sources of free gallic acid produced during fermentation, but previous research showed that the content of free phenolic compounds increases during fermentation because of microbial hydrolysis of the conjugated forms. $^{27,28)}$
We provided several lines of evidence suggesting that gallic acid is the major bioactive component in FPE. First, FPE and gallic acid effectively blocked the differentiation of 3T3-L1 adipocytes (Figs. 4, 5). Second, treatment of 3T3-L1 adipocytes with FPE and gallic acid significantly activated AMPK, which plays a central role in the regulation of glucose and lipid homeostasis (Figs. 6, 7). Third, the effects of FPE and gallic acid on 3T3-L1 adipocyte differentiation were dramatically abolished when AMPK activation was blocked by pharmacological inhibition or molecular manipulation (Figs. 6, 7). SREBP-1c is a critical transcription factor which induces adipocyte gene expression and differentiation, ${ }^{29)}$ and also activates PPAR $\gamma$ 
A
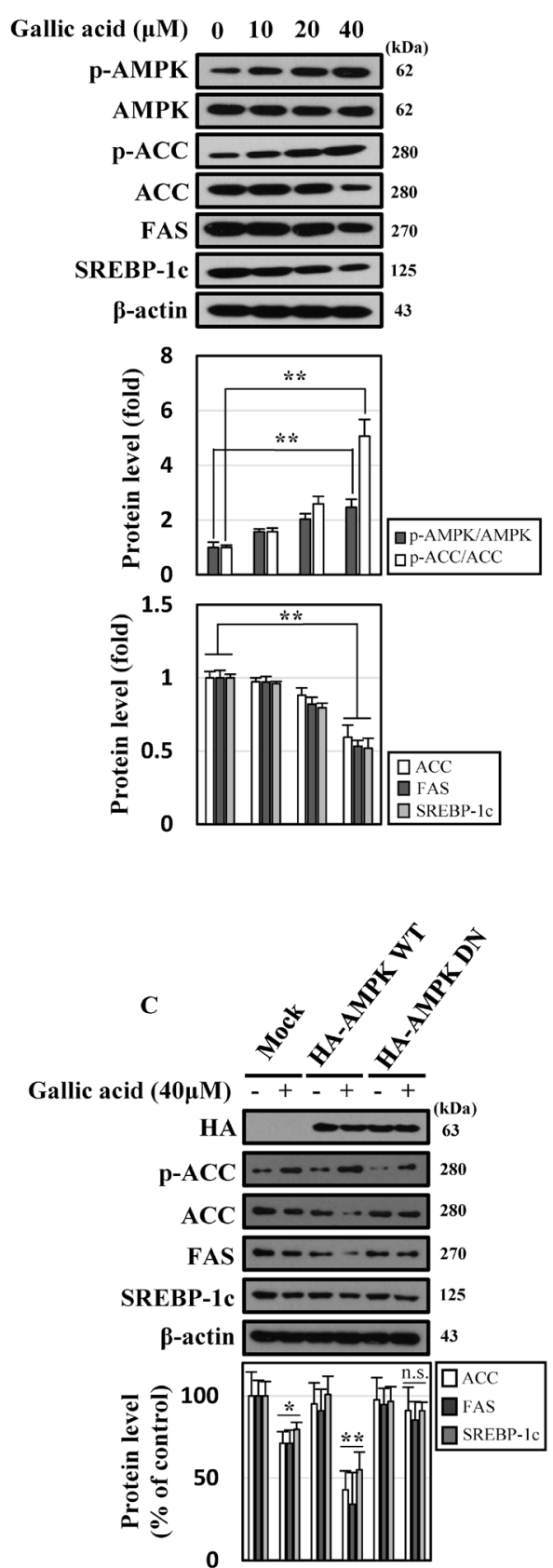

B

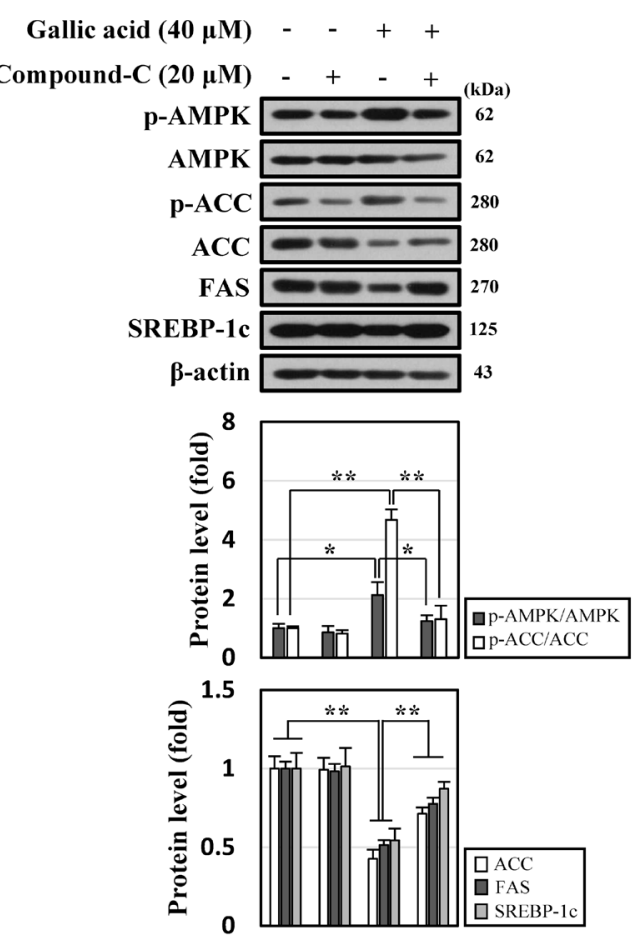

Fig. 7. Gallic Acid Inhibits the Expression of Genes Involved in Fatty Acid Synthesis by Activation of AMPK in 3T3-L1 Adipocytes

(A, B) Different concentrations of gallic acid were added into 3T3-L1 cell in the absence or presence of compound C (AMPK inhibitor). (C) 3T3-L1 cell were transfected with expression vectors for the WT or DN form of HA-tagged AMPK and then incubated with gallic acid for $24 \mathrm{~h}$. Under the conditions, Western blots were performed. Bars represent mean \pm S.D. $\left({ }^{*} p<0.05, * * p<0.01\right)$.

through production of endogenous ligand. ${ }^{30)}$ The relationship between AMPK and SREBP-1c has been suggested for a long time. It was initially reported that AMPK activation by metformin resulted in the suppression of SREBP-1c activity and amount. ${ }^{31)}$ Recently, it was demonstrated that AMPK directly phosphorylates SREBP-1c and inhibit the activity. ${ }^{32)}$ Moreover, there are several reports showing that direct pharmacological activation of AMPK inhibits adipogenesis. ${ }^{33,34)}$ Collectively, our results suggest that gallic acid is a major bioactive component in FPE, and it exerts potent anti-obesity effects in vivo and in vitro through AMPK activation. A couple of recent studies have also reported beneficial effects of gallic acid on high fat diet-induced obesity, but the underlying mechanisms including changes in genes expression involved in fatty acid synthesis are yet to be fully elucidated. ${ }^{35,36)}$ More relevant to our results, a recent report demonstrated that gallic acid regulates body weight through activation of AMPK, SIRT1, and PGC- $1 \alpha$, but the casual relationship between the weight loss and these molecules was not clearly demonstrated. ${ }^{37)}$ Gallic acid was also reported to increase glucose uptake in 3T3-L1 cells in an PI3 kinase-dependent manner without AMPK activation, ${ }^{38)}$ and in this case, it is presumed that AMPK was not activated due to low concentration and insufficient treatment time of gallic acid. 
In addition to gallic acid, several other polyphenol compounds, including resveratrol, ${ }^{39)}$ quercetin, ${ }^{40)}$ curcumin, ${ }^{41)}$ and catechins, ${ }^{42)}$ can also activate AMPK and attenuate the effects of metabolic syndrome. AMPK activation occurs due to allosteric activation by AMP accumulation as a result of ATP depletion. Upstream kinases, such as LKB1 and CaMKK, also activate AMPK. ${ }^{23-26)}$ Previous research reported that resveratrol inhibited mitochondrial ATP synthase, leading to ATP depletion, ${ }^{43-45)}$ and that curcumin decreased the ATP level in vascular smooth muscle cells. ${ }^{46)}$ Similarly, gallic acid inhibited mitochondrial respiration in acute myeloid leukemia, and contributed to the induction of apoptosis. ${ }^{47)}$ Therefore, the effect of gallic acid on mitochondrial function varies among different types of cells, and the exact mechanisms by which it activates AMPK are unknown.

In the present study, we demonstrated the anti-obesity effects of FPE occurred via AMPK activation. Activation of AMPK is beneficial for prevention of metabolic diseases, ${ }^{48,49}$ and also for suppressing oncogenesis. ${ }^{50,51)}$ Gallic acid, the major component of FPE, also prevents metabolic diseases, ${ }^{52,53)}$ and suppresses oncogenesis. ${ }^{54,55)}$ We thus hypothesize that consumption of food products with FPE may have broad effects on promoting human health.

Acknowledgments This work was supported by "Food Functionality Evaluation Program" under the Ministry of Agriculture, Food and Rural Affairs, and partly the Korea Food Research Institute, and a grant (HI14C2700) from the Korean Health Technology R\&D Project (AhR project) and a grant from the National Research Foundation of Korea (NRF2018R1D1A1B07049826).

Conflict of Interest The authors declare no conflict of interest.

\section{REFERENCES}

1) Zou B, Wu J, Yu Y, Xiao G, Xu Y. Evolution of the antioxidant capacity and phenolic contents of persimmon during fermentation. Food Sci. Biotechnol., 26, 563-571 (2017).

2) Butt MS, Sultan MT, Aziz M, Naz A, Ahmed W, Kumar N, Imran M. Persimmon (Diospyros kaki) fruit: hidden phytochemicals and health claims. EXCLI Journal., 14, 542-561 (2015).

3) Oh ST, Zheng L, Shin YK, An BK, Kang CW. Effects of dietary persimmon peel and its ethanol extract on the production performance and liver lipids in the late stage of egg production in laying hens. Asian-Australas. J. Anim. Sci., 26, 260-265 (2013).

4) Xie C, Xie Z, Xu X, Yang D. Persimmon (Diospyros kaki L.) leaves: a review on traditional uses, phytochemistry and pharmacological properties. J. Ethnopharmacol., 163, 229-240 (2015).

5) Bae UJ, Park SH, Jung SY, Park BH, Chae SW. Hypoglycemic effects of aqueous persimmon leaf extract in a murine model of diabetes. Mol. Med. Rep., 12, 2547-2554 (2015).

6) Lee YA, Cho EJ, Tanaka T, Yokozawa T. Inhibitory activities of proanthocyanidins from persimmon against oxidative stress and digestive enzymes related to diabetes. J. Nutr. Sci. Vitaminol., 53, 287-292 (2007).

7) Lee YA, Cho EJ, Yokozawa T. Effects of proanthocyanidin preparations on hyperlipidemia and other biomarkers in mouse model of type 2 diabetes. J. Agric. Food Chem., 56, 7781-7789 (2008).

8) George AP, Redpath S. Health and medicinal benefits of persimmon fruit: A review. Adv. Hortic. Sci., 22, 244-249 (2008).

9) Zou B, Li CM, Chen JY, Dong XQ, Zhang Y, Du J. High molecular weight persimmon tannin is a potent hypolipidemic in high-cholesterol diet fed rats. Food Res. Int., 48, 970-977 (2012).

10) Matsumoto K, Yokoyama S, Gato N. Hypolipidemic effect of young persimmon fruit in C57BL/6.KOR-ApoE ${ }^{\text {shl }}$ mice. Biosci. Biotechnol. Biochem., 72, 2651-2659 (2008).

11) Kadowaki A, Hashimoto N, Yokoyama S, Gato N, Matsumoto K. Persimmon fruit tannin-rich fiber reduces cholesterol levels in humans. Ann. Nutr. Metab., 62, 1-6 (2012).

12) Ge Z, Zhu W, Peng J, Deng X, Li C. Persimmon tannin regulates the expression of genes critical for cholesterol absorption and cholesterol efflux by LXR $\alpha$ independent pathway. J. Funct. Foods, 23, 283-293 (2016).

13) Matsumoto K, Watanabe Y, Ohya M, Yokoyama S. Young persimmon fruits prevent the rise in plasma lipids in a diet-induced murine obesity model. Biol. Pharm. Bull., 29, 2532-2535 (2006).

14) Hwang KA, Hwang YJ, Hwang IG, Song J, Cho SM. Cholesterollowering effect of astringent persimmon fruits (Diospyros kaki Thunb.) extracts. Food Sci. Biotechnol., 26, 229-235 (2017).

15) Liu C, Kurakane S, Takita J, Itano R, Soga T, Oikawa A, Igarashi K. Antihypertensive effects of unripe persimmon (Diospyros kaki L. cv. Hiratanenashi) fruit and its component in spontaneously hypertensive rats. Food Sci. Technol. Res., 18, 391-398 (2012).

16) Hidalgo $\mathrm{C}$, Mateo E, Mas A, Torija MJ. Identification of yeast and acetic acid bacteria isolated from the fermentation andacetification of persimmon (Diospyros kaki). Food Microbiol., 30, 98-104 (2012).

17) Zhu JC, Niu YW, Feng T, Liu SJ, Cheng HX, Xu N, Yu HY, Xiao ZB. Evaluation of the formation of volatiles and sensory characteristics of persimmon (Diospyros kaki L.f.) fruit wines using different commercial yeast strains of Saccharomyces cerevisiae. Nat. Prod. Res., 28, 1887-1893 (2014).

18) Hidalgo C, Mateo E, Cerezo AB, Torija MJ, Mas A. Technological process for production of persimmon and strawberry vinegars. Int. J. Wine Res., 2, 55-61 (2010).

19) Kim DH, Lee SB, Jeon JY, Park HD. Development of air-blast dried non-Saccharomyces yeast starter for improving quality of Korean persimmon wine and apple cider. Int. J. Food Microbiol., 290, 193204 (2019).

20) Bell V, Ferrão J, Pimentel L, Pintado M, Fernandes T. One health, fermented foods, and gut microbiota. Foods, 7, 195 (2018).

21) Haile M, Kang WH. Antioxidant activity, total polyphenol, flavonoid and tannin contents of fermented green coffee beans with selected yeasts. Fermentation, 5, 29 (2019).

22) Rashad MM, Mahmoud AE, Abdou HM, Nooman MU. Improvement of nutritional quality and antioxidant activities of yeast fermented soybean curd residue. Afr. J. Biotechnol., 10, 5504-5513 (2011).

23) Hardie DG. AMP-activated protein kinase: maintaining energy homeostasis at the cellular and whole-body levels. Annu. Rev. Nutr. 34, 31-55 (2014).

24) Ha J, Guan KL, Kim J. AMPK and autophagy in glucose/glycogen metabolism. Mol. Aspects Med., 46, 46-62 (2015).

25) Garcia D, Shaw RJ. AMPK: mechanisms of cellular energy sensing and restoration of metabolic balance. Mol. Cell, 66, 789-800 (2017).

26) Hardie DG, Ashford MLJ. AMPK: regulating energy balance at the cellular and whole body levels. Physiology, 29, 99-107 (2014).

27) Kim KS, Park KS, Kim MJ, Kim SY. Free phenolic contents and their antioxidant activities of fresh and fermented rice spent water. Food Sci. Biotechnol., 19, 1415-1420 (2010).

28) Katina K, Liukkonen KH, Kaukovirta-Norja A, Adlercreutz H, Heinonen SM, Lampi AM, Pihlava JM, Poutanen K. Fermentationinduced changes in the nutritional value of native or germinated rye. J. Cereal Sci., 46, 348-355 (2007).

29) Kim JB, Spiegelman BM. ADD1/SREBP1 promotes adipocyte differentiation and gene expression linked to fatty acid metabolism. Genes Dev., 10, 1096-1107 (1996). 
30) Kim JB, Wright HM, Wright M, Spiegelman BM. ADD1/SREBP1 activates PPAR gamma through the production of endogenous ligand. Proc. Natl. Acad. Sci. U.S.A., 95, 4333-4337 (1998).

31) Zhou G, Myers R, Li Y, Chen Y, Shen X, Fenyk-melody J, Wu M, Ventre J, Doebber T, Fujii N, Musi N, Hirshman MF, Goodyear LJ, Moller DE. Role of AMP-activated protein kinase in mechanism of metformin action. J. Clin. Invest., 108, 1167-1174 (2001).

32) Li Y, Xu S, Mihaylova MM, Zheng B, Hou X, Jiang B, Park O, Luo Z, Lefai E, Shyy JYJ, Gao B, Wierzbicki M, Verbeuren TJ, Shaw RJ, Cohen RA, Zang M. AMPK phosphorylates and inhibits SREBP activity to attenuate hepatic steatosis and atherosclerosis in diet-induced insulin-resistant mice. Cell Metab., 13, 376-388 (2011).

33) Lee H, Kang RU, Bae S, Yoon Y. AICAR, an activator of AMPK, inhibits adipogenesis via the WNT/ $\beta$-catenin pathway in 3T3-L1 adipocytes. Int. J. Mol. Med., 28, 65-71 (2011).

34) Wang L, Di L. Wnt $/ \beta$-Catenin mediates AICAR effect to increase GATA3 expression and inhibit adipogenesis. J. Biol. Chem., 290, 19458-19468 (2015).

35) Hsu CL, Yen GC. Effect of gallic acid on high fat diet-induced dyslipidaemia, hepatosteatosis and oxidative stress in rats. Br. J. Nutr., 98, 727-735 (2007).

36) Jang A, Srinivasan P, Lee NY, Song HP, Lee JW, Lee M, Jo C. Comparison of hypolipidemic activity of synthetic gallic acid-linoleic acid ester with mixture of gallic acid and linoleic acid, gallic acid, and linoleic acid on high-fat diet induced obesity in C57BL/6 Cr Slc mice. Chem. Biol. Interact., 174, 109-117 (2008).

37) Doan KV, Ko CM, Kinyua AW, Yang DJ, Choi YH, Oh IY, Nguyen NM, Ko A, Choi JW, Jeong Y, Jung MH, Cho WG, Xu S, Park KS, Park WJ, Choi SY, Kim HS, Moh SH, Kim KW. Gallic acid regulates body weight and glucose homeostasis through AMPK activation. Endocrinology, 156, 157-168 (2014).

38) Vishnu Prasad CN, Anjana T, Banerji A, Gopalakrishnapillai A. Gallic acid induces GLUT4 translocation and glucose uptake activity in 3T3-L1 cells. FEBS Lett., 584, 531-536 (2010).

39) Portillo M, Eseberri I, Fernández-Quintela A, Trepiana J, GómezZorita S, González M, Milton-Laskibar I. Involvement of 5'-activated protein kinase (AMPK) in the effects of resveratrol on liver steatosis. Int. J. Mol. Sci., 19, 3473 (2018).

40) Ahn J, Lee H, Kim S, Park J, Ha T. The anti-obesity effect of quercetin is mediated by the AMPK and MAPK signaling pathways. Biochem. Biophys. Res. Commun., 373, 545-549 (2008).

41) Miyazawa T, Nakagawa K, Kim SH, Thomas MJ, Paul L, Zingg JM, Dolnikowski GG, Roberts SB, Kimura F, Miyazawa T, Azzi A, Meydani M. Curcumin and piperine supplementation of obese mice under caloric restriction modulates body fat and interleukin-1 $\beta$. Nutr. Metab., 15, 12 (2018).

42) Li F, Gao C, Yan P, Zhang M, Wang Y, Hu Y, Wu X, Wang X,
Sheng J. EGCG reduces obesity and white adipose tissue gain partly through AMPK activation in mice. Front. Pharmacol., 9, 1366 (2018).

43) Gledhill JR, Montgomery MG, Leslie AGW, Walker JE. Mechanism of inhibition of bovine F1-ATPase by resveratrol and related polyphenols. Proc. Natl. Acad. Sci. U.S.A., 104, 13632-13637 (2007).

44) Yun H, Park S, Kim MJ, Yang WK, Im DU, Yang KR, Hong J, Choe W, Kang I, Kim SS, Ha J. Ha J. AMP-activated protein kinase mediates the antioxidant effects of resveratrol through regulation of the transcription factor FoxO1. FEBS J., 281, 4421-4438 (2014).

45) Zheng J, Ramirez VD. Inhibition of mitochondrial proton F0F1ATPase/ATP synthase by polyphenolic phytochemicals. Br. J. Pharmacol., 130, 1115-1123 (2000).

46) Grabowska W, Sikora E, Bielak-Zmijewska A, Wnuk M, Wasiak E, Lewinska A, Suszek M. Curcumin elevates sirtuin level but does not postpone in vitro senescence of human cells building the vasculature. Oncotarget, 7, 19201-19213 (2016).

47) Gu R, Zhang M, Meng H, Xu D, Xie Y. Gallic acid targets acute myeloid leukemia via Akt/mTOR-dependent mitochondrial respiration inhibition. Biomed. Pharmacother., 105, 491-497 (2018).

48) Ramesh M, Vepuri SB, Oosthuizen F, Soliman ME. Adenosine monophosphate-activated protein kinase (AMPK) as a diverse therapeutic target: a computational perspective. Appl. Biochem. Biotechnol., 178, 810-830 (2016).

49) Gasparrini M, Giampieri F, Alvarez Suarez JM, Mazzoni L, Y Forbes Hernandez T, Quiles JL, Bullon P, Battino M. AMPK as a new attractive therapeutic target for disease prevention: the role of dietary compounds AMPK and disease prevention. Curr. Drug Targets, 17, 865-889 (2016).

50) Hardie DG. AMPK: a target for drugs and natural products with effects on both diabetes and cancer. Diabetes, 62, 2164-2172 (2013).

51) Li W, Saud SM, Young MR, Chen G, Hua B. Targeting AMPK for cancer prevention and treatment. Oncotarget, 6, 7365-7378 (2015).

52) Abdel-Moneim A, Yousef AI, Abd El-Twab SM, Abdel Reheim ES, Ashour MB. Gallic acid and $p$-coumaric acid attenuate type 2 diabetes-induced neurodegeneration in rats. Metab. Brain Dis., 32, 1279-1286 (2017).

53) Bak EJ, Kim J, Jang S, Woo GH, Yoon HG, Yoo YJ, Cha JH. Gallic acid improves glucose tolerance and triglyceride concentration in diet-induced obesity mice. Scand. J. Clin. Lab. Invest., 73, 607-614 (2013).

54) Liu Z, Li D, Yu L, Niu F. Gallic acid as a cancer-selective agent induces apoptosis in pancreatic cancer cells. Chemotherapy, 58, 185-194 (2012).

55) Liao CC, Chen SC, Huang HP, Wang CJ. Gallic acid inhibits bladder cancer cell proliferation and migration via regulating fatty acid synthase (FAS). J. Food Drug Anal., 26, 620-627 (2018). 